\title{
PHASE TRANSITIONS IN A PIECEWISE EXPANDING COUPLED MAP LATTICE WITH LINEAR NEAREST NEIGHBOUR COUPLING
}

\author{
JEAN-BAPTISTE BARDET AND GERHARD KELLER
}

\begin{abstract}
We construct a mixing continuous piecewise linear map on $[-1,1]$ with the property that a two-dimensional lattice made of these maps with a linear north and east nearest neighbour coupling admits a phase transition. We also provide a modification of this construction where the local map is an expanding analytic circle map. The basic strategy is borroughed from [10, namely we compare the dynamics of the CML to those of a probabilistic cellular automaton of Toom's type, see 24] for a detailed discussion.
\end{abstract}

\section{INTRODUCTION}

The purpose of this article is to construct a continuous piecewise linear map $\tau$ on $I=[-1,1]$ such that the coupled map lattice (CML) $S_{\epsilon}: I^{\Lambda} \rightarrow I^{\Lambda}\left(\Lambda=\mathbb{Z}^{2}\right.$ or $\left.=\mathbb{Z} /(L \mathbb{Z})^{2}\right)$ defined by

$$
\left(S_{\epsilon}(\boldsymbol{x})\right)_{i}=(1-\epsilon) \tau\left(x_{i}\right)+\frac{\epsilon}{2}\left(\tau\left(x_{i+e_{1}}\right)+\tau\left(x_{i+e_{2}}\right)\right)
$$

$\left(\boldsymbol{e}_{1}, \boldsymbol{e}_{2}\right.$ are the canonical unit vectors in $\left.\Lambda\right)$ has a phase transition in the following sense: there are $0<\epsilon_{1}<\epsilon_{2}<\eta$ such that

- for $0<\epsilon \leq \epsilon_{1}$ the infinite system and also the finite ones have a unique invariant probability measure with absolutely continuous finite-dimensional conditional marginals (so the measure is absolutely continuous if $\Lambda$ is finite),

- for $\epsilon_{2} \leq \epsilon \leq \eta$ the infinite system has at least two such invariant probability measures while the finite systems still have a unique absolutely continuous invariant probability measure.

Using the notations

$$
\begin{aligned}
& \Phi_{\epsilon}: \Omega \rightarrow \Omega, \quad\left(\Phi_{\epsilon}(x)\right)_{i}=(1-\epsilon) x_{i}+\frac{\epsilon}{2}\left(x_{i+e_{1}}+x_{i+e_{2}}\right), \\
& T: \Omega \rightarrow \Omega, \quad(T \boldsymbol{x})_{i} \quad=\tau\left(x_{i}\right),
\end{aligned}
$$

we can write $S_{\epsilon}=\Phi_{\epsilon} \circ T$, and as $S_{\epsilon} \circ \Phi_{\epsilon}=\Phi_{\epsilon} \circ\left(T \circ \Phi_{\epsilon}\right)$, it is equivalent to study instead of $S_{\epsilon}$ the system

$$
T_{\epsilon}: I^{\Lambda} \rightarrow I^{\Lambda}, \quad T_{\epsilon}(\boldsymbol{x})=T\left(\Phi_{\epsilon}(\boldsymbol{x})\right) .
$$

Date: May 18, 2006.

2000 Mathematics Subject Classification. 37L40,37L60,82C20.

Key words and phrases. Coupled map lattice, piecewise expanding map, phase transition.

G.K. thanks the colleagues at the UFR Mathématiques of the University of Rennes 1 for their hospitality during his stay in March and April 2006. This note would not have been written, if both authors had not had the chance to attend the workshop "COUPLED MAP LATTICES 2004" at the IHP, Paris. Also many discussions over the years with Carlangelo Liverani helped to shape ideas. 
In order to state our results we adopt the notation of [20]: Let $\Omega=I^{\Lambda}$ and let $\mathcal{M}(\Omega)$ be the set of signed Borel measures on $\Omega .{ }^{1}$ We need to introduce the concept of measures of bounded variation. For $\mu \in \mathcal{M}(\Omega)$ let

$$
\operatorname{Var} \mu:=\sup _{i \in \Lambda} \sup _{|\varphi|_{\mathcal{C}^{0}(\Omega)} \leq 1} \mu\left(\partial_{i} \varphi\right)
$$

Here $\partial_{i}$ denotes the partial derivative with respect to $x_{i}{ }^{2}$ It is easy to prove that the set $B V_{\Omega}:=\{\mu \in \mathcal{M}(\Omega)$ : $\operatorname{Var} \mu<\infty\}$ consists of measures whose finite dimensional marginals are absolutely continuous with respect to Lebesgue and the density is a function of bounded variation [18. In addition, such measures have finite entropy density with respect to Lebesgue [19 Corollary 4.1]. In fact, "Var" is a norm and, with this norm, $B V_{\Omega}$ is a Banach space. Remark that for finite $\Lambda$ and $d \mu=f d m^{\Lambda}$ where $m$ denotes Lebesgue measure on $I, \operatorname{Var}(\mu)$ is just $\operatorname{var}(f)$ in the sense of functions of bounded variation. ${ }^{3}$

Our main result is

Theorem 1. With the piecewise linear map $\tau$ we propose in section 2 the system $\left(T_{\epsilon}, \Omega\right)$ has the following properties: There are $0<\epsilon_{1}<\epsilon_{2}<\eta$ such that the following hold:

a) For $\epsilon \in\left[0, \frac{1}{4}\right]$, the map $T_{\epsilon}$ has at least one invariant probability measure in $B V_{\Omega}$ which is also translation invariant.

b) For $\epsilon \in\left[0, \epsilon_{1}\right]$, the map $T_{\epsilon}$ has a unique invariant probability measure in $B V_{\Omega}$. (This measure is necessarily also translation invariant.)

c) For $\epsilon \in\left[\epsilon_{2}, \eta\right]$, the map $T_{\epsilon}$ has at least two invariant probability measures $\mu_{\epsilon}^{+}$ and $\mu_{\epsilon}^{-}$in $B V_{\Omega}$ with $\mu_{\epsilon}^{+}\left\{x_{0} \leq 0\right\}=\mu_{\epsilon}^{-}\left\{x_{0} \geq 0\right\}<\frac{1}{2}$.

Assertions (aid) and $\mathrm{b}$ ) follow rather directly from known results, essentially from 16] and [19], respectively. For the proof of assertion [C) we rely heavily on the construction of a phase transition in Toom's probabilistic cellular automaton (PCA) as presented in 23$]$.

The idea to link the dynamics of a CML to those of a PCA was introduced by Gielis and MacKay in [10] where they construct CMLs with simple piecewise linear Markov maps as local units and with discrete couplings in such a way that their dynamics are isomorphic to those of certain PCAs. This is definitively not the case in our model because we use a "traditional" directed nearest neighbour coupling, and so a number of additional arguments are necessary to link our CML to a PCA of Toom's type. These arguments are provided in sections 4 and 5 . On the other hand our local map $\tau$ has a very large number of monotone branches so that it acts nearly as an instantaneous local random generator unlike the rather simple local maps for which Boldrighini et al 4 exhibited phase transitions numerically even in a one-dimensional lattice. (See also earlier references given in that paper or in [12.)

We fix some further notation: For $\Lambda^{\prime} \subseteq \Lambda$ let $\mathcal{F}_{\Lambda^{\prime}}$ be the $\sigma$-algebra on $\Omega$ generated by the coordinates $x_{i}, i \in \Lambda^{\prime}$.

Definition 1. a) The probability measure $\nu$ on $\Omega$ belongs to the class $A C_{\Omega}$ if it has absolutely continuous finite-dimensional marginal distributions, i.e. if, for each

\footnotetext{
${ }^{1}$ We use the product topology on $\Omega$.

${ }^{2}$ Here and in the sequel all test functions $\varphi: \Omega \rightarrow \mathbb{R}$ depend on only finitely many coordinates and are $C^{1}$ with respect to these coordinates.

${ }^{3}$ See 19 for a careful discussion of bounded variation in the present context and the relevant associated properties.
} 
finite $\Lambda^{\prime} \subset \Lambda$, the projection of $\nu$ to $I^{\Lambda^{\prime}}$ is absolutely continuous with respect to Lebesgue measure on $I^{\Lambda^{\prime}}$.

b) The probability measure $\nu$ on $\Omega$ belongs to the class $A C C_{\Omega}$ if, for each finite $\Lambda^{\prime} \subset \Lambda, \nu$ has an $\mathcal{F}_{\Lambda \backslash \Lambda^{\prime}-\text { measurable family of conditional probability distributions }}$ on $I^{\Lambda^{\prime}}$ which are all absolutely continuous with respect to Lebesgue measure on $I^{\Lambda^{\prime}}$.

c) $B y B V_{\Omega}\left(T_{\epsilon}\right), A C_{\Omega}\left(T_{\epsilon}\right)$, and $A C C_{\Omega}\left(T_{\epsilon}\right)$ we denote the $T_{\epsilon}$-invariant measures in $B V_{\Omega}, A C_{\Omega}$, and $A C C_{\Omega}$, respectively.

Remark 1.1. We have the following two inclusions:

$$
\left\{\nu \in B V_{\Omega}: \nu \text { is a probability measure }\right\} \subseteq A C C_{\Omega} \subseteq A C_{\Omega}
$$

The second one is obvious, the first one was proved in [15, Lemma 4]. In our particular setting of one-sided directed couplings even more is known. The invariant measures belong to $A C C_{\Omega}$, namely:

$$
\left\{\nu \in B V_{\Omega}\left(T_{\epsilon}\right): \nu \text { is a probability measure }\right\}=A C C_{\Omega}\left(T_{\epsilon}\right) \subseteq A C_{\Omega}\left(T_{\epsilon}\right),
$$

see [22, Proposition 2(a)]. It follows that the unique invariant measure from Theorem [1b is indeed unique within the class $A C C_{\Omega} .{ }^{4}$ Whether this is true more generally sems to be an open question.

Our goal is to construct a coupled map lattice with a phase transition and not merely with a bifurcation at some critical parameter. As a criterion for a true phase transition in this sense MacKay [24] suggests that the dynamical system $\left(T_{\epsilon}, \Omega\right)$ should satisfy a kind of space-time specification property called indecomposability - also after the phase transition has occured. But since in our case the dynamics do not even have a tractable symbolic representation, this property seems inappropriate here. Instead we prove that each finite lattice version of the system $\left(S_{\epsilon}, \Omega\right)$ has a unique absolutely continuous invariant measure which is mixing and equivalent to the finite-dimensional Lebesgue measure on $I^{\Lambda}$. More precisely:

Theorem 2. Let $\Lambda=(\mathbb{Z} / L \mathbb{Z})^{2}$ for some $L \in \mathbb{Z}_{+}$and define $T_{\epsilon}: I^{\Lambda} \rightarrow I^{\Lambda}$ as in (1.4) with periodic boundary conditions. For the map $\tau$ from Theorem 1 and $\epsilon \in$ $\left[0, \frac{1}{4}\right]$, the system $\left(T_{\epsilon}, I^{\Lambda}\right)$ has a unique absolutely continuous invariant probability measure $d \mu_{\epsilon, \Lambda}=h_{\epsilon, \Lambda} d m^{\Lambda}$ with the following properties:

i) $\left(T_{\epsilon}, \mu_{\epsilon, \Lambda}\right)$ is mixing. Indeed, it has exponentially decreasing correlations in time for smooth observables (with a speed of decay that depends heavily on the system size $|\Lambda|$, though).

ii) The density $h_{\epsilon, \Lambda}$ is of bounded variation, so in particular $h_{\epsilon, \Lambda} \in L_{m^{\Lambda}}^{1+1 /(|\Lambda|-1)}$, and $h_{\epsilon, \Lambda}>0$ Lebesgue-almost everywhere.

The same results remain true if instead of periodic boundary conditions one prescribes fixed or free boundary conditions.

We give the proof of this theorem in section [ 6 It is a simple variant of the folklore type argument from [14].

Finally, in section 7 we discuss how the same results as above can be produced for an analytic expanding circle map $\tau$. We are not able, however, to replace also

\footnotetext{
${ }^{4}$ Of course such statements need an assumption on the coupling strength $\epsilon$. In 22 it is required that $|\epsilon|$ is "sufficiently small". What is needed for 1.7 is a suitable Lasota-Yorke inequality, and that this holds for $|\epsilon| \leq \frac{1}{4}$ follows just like our Lemma 4.1
} 


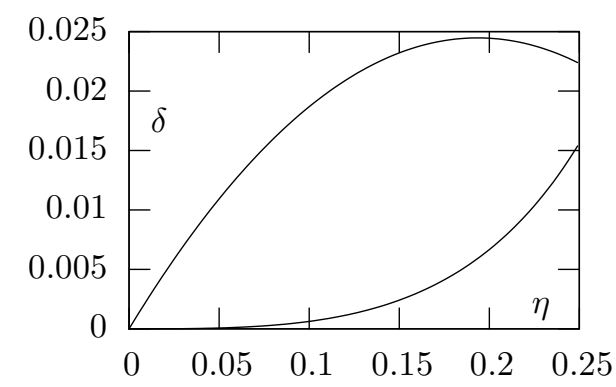

Figure 1. Admissible $(\eta, \delta)$-pairs: the region between the two curves.

the diffusive nearest neighbour coupling by a coupling that can be described by a diffeomorphism of the state space $\Omega$, which on the one hand is close enough to the identity for the existence part of Theorem 1 but on the other hand is sufficiently far away from the identity to admit a phase transition. So we are not able to construct examples of phase transitions in a class of systems as studied e.g. in [2, 3, 6, $6,8,8,9,11,25]$.

\section{THE LOCAL MAP}

The basic ingredient of the construction is a continuous piecewise linear Markov map $\tilde{\tau}:[-1,1] \rightarrow[-v, v]$ where $0<v<1$ and (for later use) $0<a<b<$ $c<d<u<v$ are suitable numbers. The map $\tilde{\tau}$ is symmetric in the sense that $\tilde{\tau}(-x)=-\tilde{\tau}(x)$, it leaves the two intervals $[-v,-c]$ and $[c, v]$ invariant, and its restrictions to each of these intervals are mixing with a strictly positive invariant density. However, the invariant density on $[c, v]$, call it $\tilde{h}$, is highly concentrated on the subinterval $[d, u] \subset[c, v]$. The map $\tau$ on $[-1,1]$ is then defined as

$$
\tau(x)=\hat{\tau}^{3}(x) \text { where } \hat{\tau}(x)=\frac{1}{v} \cdot \tilde{\tau}^{k}(x)
$$

with a suitable (rather large) $k \in \mathbb{N}$. In particular, $k$ will be chosen such that $\left|\hat{\tau}^{\prime}\right| \geq 4$.

The construction of the map $\tilde{\tau}$ depends on two parameters $\delta, \eta>0$ which obey the inequalities

$$
\eta<\frac{1}{4} \quad \text { and } \quad \frac{\eta^{3}}{2-4 \eta}<\delta<\frac{\eta^{3}-3 \eta^{2}+\eta}{4-2 \eta}
$$

The range of admissible values of $\eta$ and $\delta$ is the region between the two curves in Figure 1 One possible choice is $\eta=\frac{1}{5}$ and $\delta=\frac{1}{50}$. So we use these values in the sequel to illustrate our construction, but each other choice of $\eta$ and $\delta$ satisfying (2.2) would do as well. Figure 2 shows the graphs of $\tilde{\tau}$ and of $\left.\tilde{\tau}\right|_{[c, v]}$ that will be 

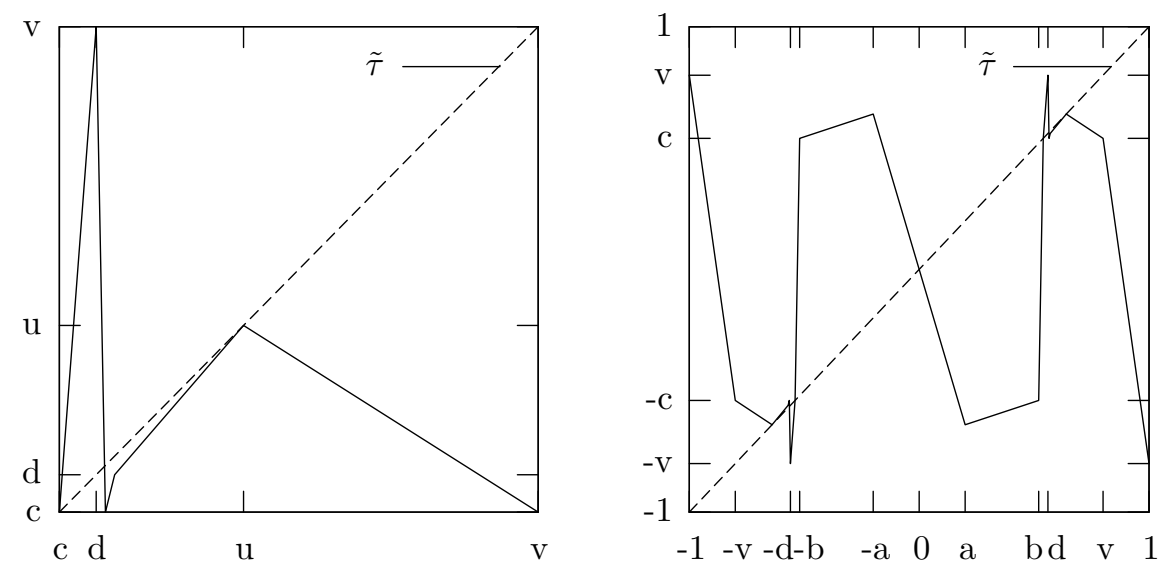

FIgURE 2. The graphs of $\tilde{\tau}$ (l.h.s.) and of $\left.\tilde{\tau}\right|_{[c, d]}$ (r.h.s.) with parameters $\eta=\frac{1}{5}$ and $\delta=\frac{1}{50}$. The parameter $\gamma$ is set to $\frac{1}{200}$ for this figure. It has to be chosen much smaller in order to construct a phase transition.

defined using these parameters. Indeed, let

$$
\begin{aligned}
& a=1-4 \eta \\
&<\quad b=1-2 \eta-4 \delta \\
&<\quad c=1-2 \eta-3 \delta \\
&<\quad d=1-2 \eta-2 \delta \\
&<\quad u=1-2 \eta+\eta^{2}=v^{2} \\
&<\quad v=1-\eta
\end{aligned}
$$

and, with a suitable (rather small) $\gamma>0$,

$$
\begin{aligned}
d & <d^{\prime}=d+\gamma \\
& <d^{\prime \prime}=d+2 \gamma \\
& <u .
\end{aligned}
$$

Observe that, by this choice,

$$
c<u=v^{2}
$$

Define $\left.\tilde{\tau}\right|_{[0,1]}$ as the piecewise linear interpolation of

$$
\begin{aligned}
& \tilde{\tau}(0)=0, \tilde{\tau}(a)=-u, \tilde{\tau}(b)=-c, \tilde{\tau}(c)=c, \tilde{\tau}(d)=v, \tilde{\tau}\left(d^{\prime}\right)=c, \\
& \tilde{\tau}\left(d^{\prime \prime}\right)=d, \tilde{\tau}(u)=u, \tilde{\tau}(v)=c, \tilde{\tau}(1)=-v,
\end{aligned}
$$

and extend $\tilde{\tau}$ to all of $[-1,1]$ by $\tilde{\tau}(-x)=-\tilde{\tau}(x)$.

By definition, $\tilde{\tau}$ and also the restriction $\left.\tilde{\tau}\right|_{[c, v]}$ are piecewise linear Markov maps, so their invariant densities can be calculated as eigenvectors of corresponding Markov matrices. The unique $\tilde{\tau}$-invariant probability density $\tilde{h}$ on $[c, v]$ is constant on 
$[c, d),[d, u]$ and on $(u, v]$. Using a computer algebra system one checks the following expressions for the corresponding probabilities under $\tilde{h}(x) d x$ :

$$
\begin{aligned}
& \int_{c}^{d} \tilde{h}(x) d x=\frac{\left(\eta^{2}+4 \delta\right)}{\eta^{4}+4 \delta \eta^{2}+4 \delta^{2}} \gamma+\mathrm{O}\left(\gamma^{2}\right) \\
& \int_{d}^{u} \tilde{h}(x) d x=1-\frac{3 \eta^{3}+10 \delta \eta+12 \delta^{2}-3 \delta \eta^{2}-2 \eta^{4}}{\eta^{5}+3 \delta \eta^{4}+4 \delta \eta^{3}+12 \delta^{2} \eta^{2}+4 \delta^{2} \eta+12 \delta^{3}} \gamma+\mathrm{O}\left(\gamma^{2}\right) \\
& \int_{u}^{v} \tilde{h}(x) d x=\frac{6 \delta \eta+2 \eta^{3}-6 \delta \eta^{2}-2 \eta^{4}}{\eta^{5}+3 \delta \eta^{4}+4 \delta \eta^{3}+12 \delta^{2} \eta^{2}+4 \delta^{2} \eta+12 \delta^{3}} \gamma+\mathrm{O}\left(\gamma^{2}\right) .
\end{aligned}
$$

(For $\eta=\frac{1}{5}$ and $\delta=\frac{1}{50}$ the corresponding values are approximately: $18.75 \gamma+\mathrm{O}\left(\gamma^{2}\right)$, $1-37.98 \gamma+\mathrm{O}\left(\gamma^{2}\right)$ and $19.23 \gamma+\mathrm{O}\left(\gamma^{2}\right)$.) Furthermore, it is easy to check that each nontrivial interval contains a point which, under iteration of $\tilde{\tau}$, eventually is mapped into $[-v,-c] \cup[c, v]$. Hence, all invariant probability densities of $\tilde{\tau}$ are convex combinations

$$
\tilde{h}_{\alpha}(x):=\alpha \tilde{h}(x)+(1-\alpha) \tilde{h}(-x) \quad(0 \leq \alpha \leq 1) .
$$

Because of (2.5), for each admissible pair $(\eta, \delta)$ and for each $\kappa>0$ one can choose $\gamma>0$ sufficiently small such that, for all $\alpha \in[0,1]$,

$$
\int_{[-u,-d] \cup[d, u]} \tilde{h}_{\alpha} d m>1-\kappa .
$$

Let $m$ be Lebesgue measure and denote by $P_{\tau}, P_{\tilde{\tau}}$ and $P_{\hat{\tau}}$ the Perron-Frobenius operators of $\tau, \tilde{\tau}$ and $\hat{\tau}$, respectively. As $\left.\tilde{\tau}\right|_{[c, v]}$ is mixing and because of (2.6), there are $C>0$ and $\rho \in(0,1)$ with the following property: for each $f \in L_{m}^{1}(I)$ there is $\alpha \in[0,1]$ such that, for all $k>0$,

$$
\int\left|\left(P_{\tilde{\tau}}^{k} f\right)-\int f d m \cdot \tilde{h}_{\alpha}\right| d m \leq C \rho^{k} \operatorname{Var}(f) .
$$

see e.g. [19, Theorem 2.1].

To proceed we need the following family of probability densities on $I$ :

$$
\hat{h}_{\alpha}(x):=v \cdot \tilde{h}_{\alpha}(v x) \quad(0 \leq \alpha \leq 1) .
$$

The following two lemmas collect all the information on $\hat{\tau}$ and $P_{\hat{\tau}}$ we need.

Lemma 2.1. The map $\hat{\tau}=\frac{1}{v} \tilde{\tau}^{k}:[-1,1] \rightarrow[-1,1]$ is surjective and mixing. Indeed, if $J$ is any maximal monotonicity interval of $\hat{\tau}$, then $\hat{\tau}^{3}(J)=[-1,1]$.

Proof. $\hat{\tau}$ is surjective because $\tilde{\tau}([-v, v])=[-v, v]$. In order to prove that the piecewise expanding map $\hat{\tau}$ is mixing, it suffices to show that $\hat{\tau}^{3}(J)=[-1,1]$ for each maximal monotonicity interval $J$ of $\hat{\tau}$, see [5]: If $J$ is a maximal monotonicity interval of $\hat{\tau}$, then it is also a maximal monotonicity interval of $\tilde{\tau}^{k}$. As

$$
-1<-v<-u<-d^{\prime}<-d<-c<-a<a<c<d<d^{\prime}<u<v<1
$$

defines a Markov partition for $\tilde{\tau}$ (all branches with respect to this partition are monotone, but not all are linear neither are all these branches maximal monotone!), $\tilde{\tau}^{k} J$ contains one of the images of the Markov intervals, i.e. one of the intervals

$$
[-c, v],[-u,-c],[-v,-c],[-c, u],[-u, u],[-u, c],[c, v],[c, u] \text {. }
$$

Each of these intervals contains at least one of $[-u,-c]$ or $[c, u]$, so we will assume from now on that $\tilde{\tau}^{k} J \supseteq[c, u]$, the other case being treated in the same way because 
of the symmetry of $\tilde{\tau}$. Then $\hat{\tau} J \supseteq\left[\frac{c}{v}, \frac{u}{v}\right]$. As $\frac{u}{v}=v$ and $\tilde{\tau}(v)=c$, we see that $\tilde{\tau} \hat{\tau} J$ contains an interval $[c, c+q]$ of length $q=\frac{u-c}{v} \cdot \frac{u-c}{v-u}=\frac{\left(\eta^{2}+3 \delta\right)^{2}}{\eta(1-\eta)^{2}}$. Choose $k$ big enough such that this interval is mapped by $\tilde{\tau}^{k-2}$ at least over the interval $[c, d]$. (For $\eta=\frac{1}{5}$ and $\delta=\frac{1}{50}$ already $k \geq 2$ would do.) Then $\tilde{\tau}^{k} \hat{\tau} J \supseteq[c, v]$, so $\hat{\tau}^{2} J \supseteq\left[\frac{c}{v}, 1\right] \supset[v, 1]$. Hence $\tilde{\tau} \hat{\tau}^{2} J \supseteq[-v, c]$, so $\tilde{\tau}^{k} \hat{\tau}^{2} \supseteq[-v, v]$ and thus $\hat{\tau}^{3} \supseteq[-1,1]$.

Lemma 2.2. Recall that $\hat{\tau}=\frac{1}{v} \tilde{\tau}^{k}$ and denote by $S: I^{s} \rightarrow I^{s}$ the s-fold direct product of $\hat{\tau}$ with itself. (We will be using the case $s=4$ later.)

a) There is a constant $\beta>0$ (whose choice depends only on $\eta, \delta$ and $\gamma$ ) such that, for each $\kappa \in\left(0, \frac{1}{2}\right)$ and sufficiently large $k$, the following estimate holds for each $f \in L_{m}^{1}\left(I^{s}\right):$

$$
\operatorname{var}\left(P_{S} f\right) \leq \kappa \operatorname{var}(f)+\beta \int|f| d m
$$

b) Given $\kappa>0$, the following holds for all sufficiently large $k$ : for each $f \in L_{m}^{1}\left(I^{s}\right)$ of bounded variation there exists $\alpha=\left(\alpha_{1}, \ldots, \alpha_{s}\right) \in[0,1]^{s}$ such that

$$
\operatorname{var}\left(P_{S} f-\int f d m \cdot \hat{h}_{\alpha}\right) \leq \kappa \operatorname{var}(f)
$$

where $\hat{h}_{\alpha}\left(x_{1}, \ldots, x_{s}\right):=\hat{h}_{\alpha_{1}}\left(x_{1}\right) \cdots \hat{h}_{\alpha_{s}}\left(x_{s}\right)$.

c) Given $\kappa>0$, for all sufficiently small $\gamma$ and all $\alpha \in[0,1]^{s}$,

$$
\int_{\left(\left[-\frac{u}{v},-\frac{d}{v}\right] \cup\left[\frac{d}{v}, \frac{u}{v}\right]\right)^{s}} \hat{h}_{\alpha} d m>1-\kappa .
$$

Proof. (2.10) is the finite-dimensional (uncoupled) Lasota-Yorke inequality, see e.g. [19] Lemma 3.2 and eq. (60)]. The other two estimates are immediate consequences.

b) This follows easily from (2.8) and (2.9), see e.g. [19, section 4.5].

c) This is an immediate consequence of (2.9) and (2.7) (where the $\kappa$ from (2.12) equals $1-(1-\kappa)^{s}$ in terms of the $\kappa$ from (2.7)).

Hence, the "local units" $\tau=\hat{\tau}^{3}$ will behave like Markov chains that admit transitions from the positive to the negative part of $[-1,1]$ and vice versa only with very small probabilities because, for sufficiently large $k$, most of the mass will be concentrated on $\left[-\frac{u}{v},-\frac{d}{v}\right] \cup\left[\frac{d}{v}, \frac{u}{v}\right] \subset[-v,-c] \cup[c, v]$ after each application of $\hat{\tau}$, and points in this set will not change sign during the next application of $\hat{\tau}$. Nevertheless, there is always a small mass, proportional to $\gamma$, which is mapped under the next application of $\tilde{\tau}$ to $[-v,-c]$, so transitions are never completely excluded.

In the next section we will see how coupling with at least one neighbour of the same sign can prevent such a sign flip, and we will be careful enough to make sure that coupling with two neighbours of opposite sign indeed forces a flip.

Remark 2.3. In the rest of this section and in sections 3- 5, only those properties of $\hat{\tau}$ will be used that are formulated in Lemma 2.2. (Lemma 2.1 will play a role only in the proof of Theorem [2 in section [6.) Therefore, the same proof of Theorem 1$]$ will work for two kinds of modifications of $\hat{\tau}$.

(A) All arguments remain unchanged, if we replace the decreasing branches of $\tilde{\tau}$ by increasing ones with the same domain and the same range: we obtain a piecewise linear Markov map $\dot{\tau}$, it satisfies the Lasota-Yorke inequality with 
the same constants, and it has the same associated Markov chain as $\tilde{\tau}$. So it has in particular the same 2-dimensional space of invariant densities $\tilde{h}_{\alpha} \cdot \tau$ is of course no longer continuous, but the continuity of $\tilde{\tau}$ was not used in the proof of Lemma 2.2.

(B) Let $\check{\tau}=\frac{1}{v} \dot{\tau}^{k}$. We may also replace $\check{\tau}$ by any map $\bar{\tau}$ which has full branches and whose Perron-Frobenius operator is a small perturbation of that of $\check{\tau}$ in the sense that it satisfies a Lasota-Yorke inequality with the same constants as $\hat{\tau}$ and $\check{\tau}$ (thus leading to the same choices of $\beta$ and $k=k(\kappa)$ in Lemma 2.9a) and that

$$
||\left|P_{\bar{\tau}}-P_{\check{\tau}}\right|||:=\sup \left\{\int\left|\left(P_{\bar{\tau}}-P_{\check{\tau}}\right)(f)\right| d m: \operatorname{Var}(f) \leq 1\right\}
$$

is sufficiently small. For such maps, the conclusions of Lemma 2. Sa hold trivially, and the persistence of the conclusions of Lemma 2.9.6 is a consequence of the spectral stability theorem from [17], see also [1, Sections 3.1 - 3.3] for a coherent account of the spectral (perturbation) theory for Perron-Frobenius operators of piecewise expanding transformations.

In section 7 we will come back to these remarks.

\section{The Coupling: LOCAL EFFECTS}

The coupling map $\Phi_{\epsilon}: \Omega \rightarrow \Omega$ can be described in terms of a local coupling rule $\phi_{\epsilon}: I^{3} \rightarrow I$,

$$
\begin{aligned}
\phi_{\epsilon}(x, y, z) & :=(1-\epsilon) x+\frac{\epsilon}{2}(y+z), \text { so that } \\
\left(\Phi_{\epsilon}(\boldsymbol{x})\right)_{i} & =\phi_{\epsilon}\left(\boldsymbol{x}_{\boldsymbol{i}}, \boldsymbol{x}_{\boldsymbol{i}+\boldsymbol{e}_{1}}, \boldsymbol{x}_{\boldsymbol{i}+\boldsymbol{e}_{2}}\right) .
\end{aligned}
$$

Suppose now that $x, y, z \in[-1,1], \frac{d}{v} \leq|x|,|y|,|z| \leq \frac{u}{v}=v$, and $\epsilon \in[0, \eta]$. Denote $x^{\prime}=\phi_{\epsilon}(x, y, z)$. By symmetry we may assume without loss of generality that $x>0$. Our first observation is obvious,

$$
x^{\prime} \leq v
$$

Suppose that $y>0$ or $z>0$ : Without loss of generality let $y>0$. Then

$$
x^{\prime} \geq(1-\eta) \frac{d}{v}+\frac{\eta}{2} \frac{d-u}{v}=d+\frac{\eta\left(\eta^{2}+2 \delta\right)}{2(1-\eta)}>c
$$

where we used $\delta>\frac{\eta^{3}}{2-4 \eta}$ for the last inequality, see (2.2).

Suppose that $y, z<0$ and $\epsilon=\eta$ : Then

$$
x^{\prime} \geq(1-\eta) \frac{d}{v}-\eta v=d-\eta v>a
$$

where we used for the last equation that $\delta<\frac{\eta^{3}-3 \eta^{2}+\eta}{4-2 \eta} \leq \frac{\eta+\eta^{2}}{2}$ (for $\eta \in$ $[0,1])$, see also (2.2). In this case we also have

$$
x^{\prime} \leq(1-\eta) v-\eta \frac{d}{v}=(1-\eta)^{2}-\frac{\eta(1-2 \eta-2 \delta)}{1-\eta}<b
$$


where we used again $\delta<\frac{\eta^{3}-3 \eta^{2}+\eta}{4-2 \eta}$ for the last inequality. We finally notice that inequalities (3.4) and (3.5) are strict, hence still satisfied for values of $\epsilon$ which are smaller than $\eta$, but close to it, say for $\epsilon \in\left[\epsilon_{2}, \eta\right]$.

We summarize these observations:

- If at least one of $y$ and $z$ is in $\left[\frac{d}{v}, v\right]$, then $x^{\prime} \in(c, v]$ and hence $\tilde{\tau}^{k} x^{\prime} \in[c, v]$.

- If both $y$ and $z$ are in $\left[-v,-\frac{d}{v}\right]$ and if $\epsilon \in\left[\epsilon_{2}, \eta\right]$, then $x^{\prime} \in(a, b)$ so that $\tilde{\tau}\left(x^{\prime}\right) \in[-u,-c]$ and hence $\tilde{\tau}^{k} x^{\prime} \in[-v,-c]$.

Indeed, we will see that, with very high probability, $\frac{d}{v} \leq\left|\hat{\tau}^{3}\left(x^{\prime}\right)\right| \leq v$ again so that, if $x$ is the state of the coupled system at a given time at lattice site $i$ and if $y$ and $z$ are the states at the same times at sites $i+\boldsymbol{e}_{1}$ and $i+\boldsymbol{e}_{2}$, then the sign of $x^{\prime}$, which is the updated value at site $\boldsymbol{i}$, deviates only with small probability from the "majority vote" of $x, y$ and $z$, i.e. from $\operatorname{sign}(\operatorname{sign} x+\operatorname{sign} y+\operatorname{sign} z)$. Hence this interaction mimics the local rule of Toom's probabilistic cellular automaton 27. In the next section we will see how the proof that this automaton admits at least two invariant measures can be modified for our purpose. We follow the treatment of 23$]$.

\section{The COUpling: global EFFects}

It is useful to introduce the usual total variation norm on signed measures:

$$
|\mu|:=\sup _{|\varphi|_{\mathcal{C}^{0}(\Omega)} \leq 1} \mu(\varphi) .
$$

Just like in [19, Sect. 3.3] one checks easily that

$$
|\mu| \leq \frac{1}{2} \operatorname{Var} \mu \text {. }
$$

We consider the dynamics acting directly on the measures via the linear operator $T_{\epsilon}^{*} \mu(A):=\mu\left(T_{\epsilon}^{-1} A\right)$ (for each measurable set $A$ ). The basic facts concerning the operator $T_{\epsilon}^{*}$ are detailed in the following lemma.

Lemma 4.1 (Lasota-Yorke inequality). Recall that $\hat{\tau}=\left(\frac{1}{v} \tilde{\tau}^{k}\right)$. There exists constants $k_{0}>0$ and $B>0$ which depend only on the parameters $\eta, \delta$ and $\gamma$ such that, for all integers $k \geq k_{0},\left|\hat{\tau}^{\prime}\right| \geq 4$ and such that, for each $|\epsilon| \leq \frac{1}{4}$, the operator $T_{\epsilon}^{*}$ is well defined as an operator on $B V_{\Omega}$. In addition, for each $\mu \in B V_{\Omega}$ holds true

$$
\begin{aligned}
\left|T_{\epsilon}^{*} \mu\right| & \leq|\mu| \\
\operatorname{Var}\left(T_{\epsilon}^{* n} \mu\right) & \leq 2^{-n} \operatorname{Var} \mu+B|\mu| .
\end{aligned}
$$

In particular, $\operatorname{Var}\left(T_{\epsilon}^{*} \mu\right) \leq 2 B$ for each probability measure $\mu \in B V_{\Omega}$ with $\operatorname{Var} \mu \leq$ $2 B$, and $\operatorname{Var} \mu \leq B$ for each $T_{\epsilon}$-invariant probability measure $\mu \in B V_{\Omega}$.

The same assertions hold for the case of a finite lattice $\Lambda=(\mathbb{Z} / L \mathbb{Z})^{2}$.

Proof. This follows from the special case $\theta=1$ of Proposition 4.1 in 19 . (For finite $\Lambda$ use [19, Proposition 3.2].) Observe that the proof given there for $\Lambda=\mathbb{Z}$ applies (only if $\theta=1$ !) without changes to $\Lambda=\mathbb{Z}^{2}$. The particular constants in the present lemma follow from the proofs in [19, section 3] by observing the following facts:

$\triangleright$ Our coupling is a $(1,0)$-coupling in the sense of [19] section 3.1].

$\triangleright$ We consider the local map $\tau$ itself, so $\ell=1$ in [19, section 3.4]. 
$\triangleright$ Recall that $\tau(x)=\hat{\tau}^{3}(x)=\left(\frac{1}{v} \tilde{\tau}^{k}\right)^{3}(x)$. We choose $k$ so large that $\left|\hat{\tau}^{\prime}\right| \geq 4$. An elementary detailed analysis of the map shows that this is always possible because $\left|\left(\left.\tilde{\tau}^{2}\right|_{[c, v]}\right)^{\prime}\right| \geq \min \left\{9,\left(\frac{2 \delta+\eta^{2}}{2 \delta+\eta^{2}-3 \gamma}\right)^{2}, \frac{3 \delta}{\gamma}\right\}$. Indeed, since $\gamma$ is rather small, the most critical slope of $\hat{\tau}$ occurs in a small one-sided left neighbourhood of $-a$, which is mapped to a one-sided left neighbourhood of the fixed point $u$. This slope is $\frac{\eta^{2}+3 \delta}{2 \eta-4 \delta}\left(\frac{2 \delta+\eta^{2}}{2 \delta+\eta^{2}-3 \gamma}\right)^{k-1}$. With these choices, Lemmas 3.2 and 3.3 in [19] yield the claimed values for the constants when choosing $\epsilon_{1}=\frac{1}{4}$ there. Observe also that the exponential factor $2^{-n}$ can be replaced by any factor $\rho^{n}, \rho \in(0,1)$, at the price of larger $k$ and $B$.

Remark 4.2. The choice of $k$ we make in Lemma 4.1 is sufficient for the proof of Theorem [1] For Theorem [2 our proof will require to choose a still larger $k$ : we require that $\left|\hat{\tau}^{\prime}\right| \geq \frac{12 v}{u-c}$, which implies in particular that $\left|\hat{\tau}^{\prime}\right| \geq 4$.

Proof of Theorem [1] The existence of invariant probability measures in $B V_{\Omega}$ follows from Lemma 4.1 just as in [19, section 4.4]. The proof given there for the lattice $\Lambda=\mathbb{Z}$ applies without changes to the lattice $\Lambda=\mathbb{Z}^{2}$.

Proof of Theorem [1]b This follows at once from [20].

Proof of Theorem [1] In order to get close to the formal setting of [23, we introduce the short hand notation

$$
\boldsymbol{x}^{t}:=T_{\epsilon}^{t}(\boldsymbol{x}) \quad \text { for } \boldsymbol{x} \in \Omega \text { and } t \in \mathbb{N} .
$$

In 23], the authors work with time indexed by $t \in \mathbb{Z}$, in particular they specify certain events at times $-N$ and 0 . In our setting it seems more natural to shift these events to times 0 and $N$, and since all estimates are for fixed $N$, this is just a matter of convenience.

In order to make clear which estimates we need, we reproduce here a sketch of the basic argument in 23 with notations adapted to our setting. Fix $N \in \mathbb{N}$ and let

$$
\Sigma_{+}^{N}:=\left\{\sigma=\left(\sigma_{i}^{t}\right) \in\{-1,+1\}^{\Lambda \times\{0, \ldots, N\}}: \sigma_{\mathbf{0}}^{N}=-1 \text { and } \sigma_{i}^{0}=+1 \forall \boldsymbol{i} \in \Lambda\right\}
$$

In [23. Appendix A] the authors construct a family $\mathcal{V}^{(N)}$ of nonempty finite subsets $\hat{V} \subset \Lambda \times \mathbb{N}$ together with a map $V^{(N)}: \Sigma_{+}^{N} \rightarrow \mathcal{V}^{(N)}$ in such a way that ${ }^{5}$

(i) $\# \mathcal{V}_{M}^{(N)} \leq(48)^{8 M}$ for each $M \geq 1$ where $\mathcal{V}_{M}^{(N)}:=\left\{\hat{V} \in \mathcal{V}^{(N)}: \# \hat{V}=M\right\}$.

(ii) Let $\sigma \in \Sigma_{+}^{N}$. Each $(i, t) \in V^{(N)}(\sigma)$ is an error site in space-time for the configuration $\sigma$, i.e. $\sigma_{i}^{t+1} \neq \operatorname{sign}\left(\sigma_{i}^{t}+\sigma_{i+e_{1}}^{t}+\sigma_{i+e_{2}}^{t}\right)$. In other words, $\sigma$ deviates from Toom's deterministic majority rule at $(\boldsymbol{i}, t)$.

Now we apply this purely combinatorial construction to our problem. Let

$$
\Omega_{+}:=\left\{\boldsymbol{x} \in \Omega: x_{\boldsymbol{i}}^{0}=x_{\boldsymbol{i}}>0 \forall \boldsymbol{i} \in \Lambda\right\} \text { and } \Omega_{+}^{N}:=\left\{\boldsymbol{x} \in \Omega_{+}: x_{\mathbf{0}}^{N} \leq 0\right\} .
$$

\footnotetext{
${ }^{5}$ More correctly, what we state here is a consequence of what is proved in 23 . It is precisely what we need for our proof, and a reader who attempts to go through the proof in 23] will find it a good warm-up exercise to check that our claims (i) and (ii) are indeed immediately implied by what is proved there.
} 
To each $\boldsymbol{x} \in \Omega_{+}^{N}$ we associate $\sigma(\boldsymbol{x}) \in \Sigma_{+}^{N}$ defined by $\sigma_{i}^{t}=+1$ or -1 according to whether $x_{i}^{t}>0$ or $x_{i}^{t} \leq 0$, respectively. (We do not claim that each $\sigma \in \Sigma_{+}^{N}$ occurs as some $\sigma(\boldsymbol{x})$.) For a finite subset $W$ of $\Lambda \times \mathbb{N}$ let

$$
E(W):=\left\{\boldsymbol{x} \in \Omega_{+}: \text {Each }(\boldsymbol{i}, t) \in W \text { is an error site for } \sigma(\boldsymbol{x})\right\} .
$$

Then, if $\mu$ is any probability measure on $\Omega_{+}$,

$$
\begin{aligned}
\mu\left(\Omega_{+}^{N}\right) & =\sum_{M=1}^{\infty} \sum_{\hat{V} \in \mathcal{V}_{M}^{(N)}} \mu\left\{\boldsymbol{x} \in \Omega_{+}^{N}: V^{(N)}(\sigma(\boldsymbol{x}))=\hat{V}\right\} \\
& \leq \sum_{M=1}^{\infty} \sum_{\hat{V} \in \mathcal{V}_{M}^{(N)}} \mu(E(\hat{V})) \\
& \leq \sum_{M=1}^{\infty}(48)^{8 M} \cdot q_{M}
\end{aligned}
$$

where

$$
q_{M}:=\sup \left\{\mu(E(\hat{V})): N \geq 0 \text { and } \hat{V} \in \mathcal{V}_{M}^{(N)}\right\} .
$$

In the next section we will derive the exponential estimate

$$
q_{M} \leq \Delta^{M} \text { with } \Delta=\frac{1}{4}(48)^{-8}>0
$$

valid when $\mu=\lambda_{+}$is the product Lebesgue measure on $\left[\frac{d}{v}, v\right]^{\Lambda}$. Hence, for all $N>0$,

$$
\left(T_{\epsilon}^{* N} \lambda_{+}\right)\left\{\boldsymbol{x} \in \Omega: x_{\mathbf{0}} \leq 0\right\}=\lambda_{+}\left(\Omega_{+}^{N}\right) \leq \frac{1}{3} .
$$

Now, as in [19, proof of Theorem 4.1] it follows that $\frac{1}{n} \sum_{N=1}^{n} T_{\epsilon}^{* N} \lambda_{+}$converges weakly to a $T_{\epsilon}$-invariant probability measure $\mu_{+} \in B V_{\Omega}$. So $\mu_{+}\left\{\boldsymbol{x} \in \Omega: x_{\mathbf{0}} \leq 0\right\} \leq$ $\frac{1}{3}$. Interchanging the roles of + and - one also finds a $T_{\epsilon}$-invariant probability $\mu_{-} \in B V_{\Omega}$ with $\mu_{-}\left\{\boldsymbol{x} \in \Omega: x_{\mathbf{0}} \geq 0\right\} \leq \frac{1}{3}$. This finishes the proof of Theorem [1] except for the estimate (4.7) which is derived in the next section.

\section{The exponential estimate (4.7)}

Let $U(\boldsymbol{i})=\left\{\boldsymbol{i}, \boldsymbol{i}+\boldsymbol{e}_{1}, \boldsymbol{i}+\boldsymbol{e}_{2}, \boldsymbol{i}+\boldsymbol{e}_{1}+\boldsymbol{e}_{2}\right\}$. Then $x_{\boldsymbol{i}}^{t+1}$ does not depend on $x_{\boldsymbol{j}}^{t}$ if $\boldsymbol{j} \notin U(\boldsymbol{i})$, and so no $x_{\boldsymbol{k}}^{s}$ influences any $x_{j}^{t}$ as long as $\boldsymbol{k} \in U(\boldsymbol{i})$ and $\boldsymbol{j} \in \Pi(\boldsymbol{i})$ where

$$
\Pi(\boldsymbol{i}):=\left\{\boldsymbol{j} \in \Lambda: j_{1} \geq i_{1}+2 \text { or } j_{2} \geq i_{2}+2\right\} .
$$

Hence $T_{\epsilon}$ can be "restricted" to $I^{\Pi(i)}$ as an autonomous dynamical system, call it $T_{\epsilon, \Pi(i)}$, and also to $I^{U(i) \cup \Pi(i)}$, call this $T_{\epsilon, U(i) \cup \Pi(i)}$. Observe that $T_{\epsilon, U(i) \cup \Pi(i)}$ is indeed a skew product transformation over the base $T_{\epsilon, \Pi(i)}$. Therefore, the action of $T_{\epsilon}$ on the coordinates $x_{k}, k \in U(i)$, can be interpreted as a nonautonomous dynamical system on $I^{U(i)}$ that is governed by the $T_{\epsilon, \Pi(i)}$-orbit of $\boldsymbol{x}_{\Pi(i)}$. We use the following notation:

$$
T_{\epsilon, U(i) \cup \Pi(i)}^{n}\left(y, \boldsymbol{x}_{\Pi(i)}\right)=:\left(T_{\epsilon \mid x_{\Pi(i)}^{n}}^{n}(y), T_{\epsilon, \Pi(i)}^{n}\left(\boldsymbol{x}_{\Pi(i)}\right)\right) \text { for } y \in I^{U(i)} \text { and } \boldsymbol{x}_{\Pi(i)} \in I^{\Pi(i)},
$$


and we write $P_{\epsilon \mid x_{\Pi(i)}}^{n}$ for the Perron-Frobenius operator of $T_{\epsilon \mid x_{\Pi(i)}}^{n}$ on $L_{\mathrm{Leb}}^{1}\left(I^{U(i)}\right)$. This is the setting studied in [22]. In particular, for $f \in L_{\mathrm{Leb}}^{1}\left(I^{U(i)}\right)$ and each $\boldsymbol{x}_{\Pi(i)}$,

$$
\operatorname{var}\left(P_{\epsilon \mid x_{\Pi(i)}}^{n} f\right) \leq 2^{-n} \operatorname{var}(f)+B\|f\|_{1},
$$

where, as in the proof of Lemma 4.1 the factor $2^{-n}$ is achieved by choosing $k$ in the definition of $\hat{\tau}=\frac{1}{v} \tilde{\tau}^{k}$ sufficiently large.

Now fix some $\hat{V} \in \mathcal{V}_{M}^{(N)}$. As

$$
\Lambda \times \mathbb{N}=\left(2 \Lambda \cup\left(2 \Lambda+e_{1}\right) \cup\left(2 \Lambda+\boldsymbol{e}_{2}\right) \cup\left(2 \Lambda+\boldsymbol{e}_{1}+\boldsymbol{e}_{2}\right)\right) \times(2 \mathbb{N} \cup(2 \mathbb{N}+1))
$$

is the disjoint union of eight sublattices, the intersection of $\hat{V}$ with at least one of these sublattices, call this intersection $\tilde{V}$, satisfies

$$
\# \tilde{V} \geq \frac{1}{8} \# \hat{V}=\frac{M}{8} .
$$

We are going to prove that

$$
\mu(E(\tilde{V})) \leq \Delta^{8 \cdot \# \tilde{V}} .
$$

As $E(\hat{V}) \subseteq E(\tilde{V})$, estimate (4.7) then follows at once.

In order to prove (5.4) by induction we introduce, for each $\Gamma \subseteq \Lambda$, the set

$$
\tilde{V}_{\Gamma}:=\tilde{V} \cap(\Gamma \times \mathbb{N}) .
$$

Denote also, for the moment, $\Lambda^{\prime}:=\left\{\boldsymbol{i} \in \Lambda: \tilde{V}_{\{i\}} \neq \emptyset\right\}$. By construction of $\tilde{V}$ from $\hat{V}$, we have $\Lambda^{\prime} \cap U(\boldsymbol{i})=\Lambda^{\prime} \cap\{\boldsymbol{i}\}$ for each $i \in \Lambda^{\prime}$. It follows that there is (at least) one "maximal" site $\boldsymbol{i} \in \Lambda^{\prime}$ in the sense that $\Lambda^{\prime} \backslash\{\boldsymbol{i}\} \subseteq \Pi(\boldsymbol{i})$. We fix such a site $\boldsymbol{i}$ now. In order to prove (5.4) it suffices (by induction) to show that

$$
\mu(E(\tilde{V})) \leq \Delta^{8 \cdot \# \tilde{V}_{\{i\}}} \cdot \mu\left(E\left(\tilde{V}_{\Pi(i)}\right)\right) .
$$

We may write $E(\tilde{V})=E\left(\tilde{V}_{\{i\}}\right) \cap E\left(\tilde{V}_{\Pi(i)}\right)$, where $E\left(\tilde{V}_{\Pi(i)}\right)$ only depends on coordinates at sites from $\Pi(\boldsymbol{i})$ whereas $E\left(\tilde{V}_{\{\boldsymbol{i}\}}\right)$ depends on those from $U(\boldsymbol{i}) \cup \Pi(\boldsymbol{i})$. Hence, for any $\mu \in A C C_{\Omega}$, denoting $h_{x_{\Pi(i)}}$ its conditional density on $I^{U(i)}$ given $\boldsymbol{x}_{\Pi(i)}$ outside, one has

$$
\begin{aligned}
\mu(E(\tilde{V})) & =\int_{E\left(\tilde{V}_{\Pi(i)}\right)} \int 1_{E\left(\tilde{V}_{\{i\}}\right)}\left(x_{U(i)}, x_{\Pi(i)}\right) h_{x_{\Pi(i)}}\left(x_{U(i)}\right) d x_{U(i)} d \mu\left(x_{\Pi(i)}\right) \\
& \leq \sup _{x_{\Pi(i)} \in I^{\Pi(i)}}\left(\int_{B_{0}\left(x_{\Pi(i)}\right)} h_{x_{\Pi(i)}}(y) d y\right) \cdot \mu\left(E\left(\tilde{V}_{\Pi(i)}\right)\right)
\end{aligned}
$$

with

$$
B_{0}\left(\boldsymbol{x}_{\Pi(i)}\right)=\left\{y \in I^{U(i)}:\left(y, \boldsymbol{x}_{\Pi(i)}\right) \in E\left(\tilde{V}_{\{i\}}\right)\right\} .
$$

We can now fix $\boldsymbol{x}_{\Pi(i)}$ and work with the nonautonomous system $T_{\epsilon \mid x_{\Pi(i)}}^{t}$ on $I^{U(i)}$. We also denote, for $y \in I^{U(i)}$,

$$
\Phi_{\epsilon}\left(y, \boldsymbol{x}_{\Pi(i)}^{t}\right)=:\left(\Phi_{\epsilon}^{\{t\}}(y), \Phi_{\epsilon, \Pi(i)}\left(\boldsymbol{x}_{\Pi(i)}^{t}\right)\right),
$$

see also (4.3), and, with a slight abuse of notation, we denote by $\hat{T}$ (resp. $\tilde{T}$ ) the 4 -fold direct product of $\tilde{\tau}$ (resp. $\hat{\tau}$ ) on $I^{U(i)}$. 
Let $\xi \in I^{U(i)}$. We denote $\xi^{t}=T_{\epsilon \mid x_{\Pi(i)}}^{t}(\xi)$ and

$$
\begin{aligned}
M\left(\xi^{t}\right): & =\left\{y \in I^{U(i)}: y_{i} \text { obeys the majority rule relative to } \xi^{t}\right\} \\
& =\left\{y \in I^{U(i)}: \operatorname{sign}\left(y_{i}\right)=\operatorname{sign}\left(\operatorname{sign}\left(\xi_{i}^{t}\right)+\operatorname{sign}\left(\xi_{i+e_{1}}^{t}\right)+\operatorname{sign}\left(\xi_{i+e_{2}}^{t}\right)\right)\right\} .
\end{aligned}
$$

We want to check precisely which condition on $\xi^{t}$ ensures that $\xi^{t+1} \in M\left(\xi^{t}\right)$, i.e. that $(\boldsymbol{i}, t)$ is not an error site for $\left(\xi, \boldsymbol{x}_{\Pi(i)}\right)$. We know from section 3 that if

$$
\xi^{t} \in G:=\left\{y \in I^{U(i)}:\left|y_{i}\right|,\left|y_{i+e_{1}}\right| \text { and }\left|y_{i+e_{2}}\right| \in\left[\frac{d}{v}, v\right]\right\},
$$

then $\hat{T} \Phi_{\epsilon}^{\{t\}}\left(\xi^{t}\right) \in M\left(\xi^{t}\right)$. If we assume further that $\hat{T} \Phi_{\epsilon}^{\{t\}}\left(\xi^{t}\right) \in G$, then the fact that $\tilde{\tau}^{k}\left(\left[\frac{d}{v}, v\right]\right) \subset \tilde{\tau}^{k}([c, v])=[c, v]$ implies that also $\hat{T}^{2} \Phi_{\epsilon}^{\{t\}}\left(\xi^{t}\right) \in M\left(\xi^{t}\right)$ and finally, by the same argument, we have $\hat{T}^{3} \Phi_{\epsilon}^{\{t\}}\left(\xi^{t}\right) \in M\left(\xi^{t}\right)$ provided also $\hat{T}^{2} \Phi_{\epsilon}^{\{t\}}\left(\xi^{t}\right) \in G$. This can be resumed by

$$
\begin{aligned}
E_{(i, t)}: & =\left\{\xi \in I^{U(i)}:(\boldsymbol{i}, t) \text { is an error site for }\left(\xi, \boldsymbol{x}_{\Pi(i))}\right\}\right. \\
& \subseteq\left\{\xi: \xi^{t} \notin G\right\} \cup\left\{\xi: \hat{T} \Phi_{\epsilon}^{\{t\}}\left(\xi^{t}\right) \notin G\right\} \cup\left\{\xi: \hat{T}^{2} \Phi_{\epsilon}^{\{t\}}\left(\xi^{t}\right) \notin G\right\} .
\end{aligned}
$$

This description will be used to estimate (uniformly in $\boldsymbol{x}_{\Pi(\boldsymbol{i})}$ ) the integral from (5.7). Let us assume for the moment that $t_{1}=\min \left\{t:(\boldsymbol{i}, t) \in \tilde{V}_{\{\boldsymbol{i}\}}\right\} \geq 1$ and define

$$
\begin{gathered}
\tilde{V}_{\{i\}}^{\left(t_{1}\right)}=\left\{\left(\boldsymbol{i}, t-t_{1}-1\right): t>t_{1} \text { and }(\boldsymbol{i}, t) \in \tilde{V}_{\{i\}}\right\} \\
B_{1}\left(\boldsymbol{x}_{\Pi(i)}\right)=\left\{y \in I^{U(i)}:\left(y, T_{\epsilon, \Pi(i)}^{t_{1}+1}\left(\boldsymbol{x}_{\Pi(i)}\right)\right) \in E\left(\tilde{V}_{\{i\}}^{\left(t_{1}\right)}\right)\right\}
\end{gathered}
$$

We have then $B_{0}\left(\boldsymbol{x}_{\Pi(i)}\right)=E_{\left(i, t_{1}\right)} \cap T_{\epsilon \mid x_{\Pi(i)}}^{-\left(t_{1}+1\right)} B_{1}\left(\boldsymbol{x}_{\Pi(i)}\right)$, which allows us to write, for each $h_{0}: I^{U(i)} \rightarrow[0, \infty)$ of bounded variation,

$$
\begin{aligned}
\int_{B_{0}\left(x_{\Pi(i)}\right)} h_{0}(y) d y & \\
= & \int_{B_{1}\left(x_{\Pi(i)}\right)} P_{\epsilon \mid x_{\pi(i)}}^{t_{1}+1}\left(1_{E_{\left(i, t_{1}\right)}}(y) h_{0}(y)\right) d y \\
\leq & \int_{B_{1}\left(x_{\Pi(i)}\right)} P_{\hat{T}}\left(P_{\hat{T}}^{2} P_{\Phi_{\epsilon}^{\left\{t_{1}\right\}}}\left(1_{G^{c}} P_{\hat{T}}\left(P_{\hat{T}}^{2} P_{\Phi_{\epsilon}^{\left\{t_{1}-1\right\}}} P_{\epsilon \mid x_{\pi(i)}}^{t_{1}-1} h_{0}\right)\right)\right)(y) d y \\
& +\int_{B_{1}\left(x_{\Pi(i)}\right)} P_{\hat{T}}\left(P_{\hat{T}}\left(1_{G^{c}} P_{\hat{T}}\left(P_{\Phi_{\epsilon}^{\left\{t_{1}\right\}}} P_{\epsilon \mid x_{\pi(i)}}^{t_{1}} h_{0}\right)\right)\right)(y) d y \\
& +\int_{B_{1}\left(x_{\Pi(i)}\right)} P_{\hat{T}}\left(1_{G^{c}} P_{\hat{T}}\left(P_{\hat{T}} P_{\Phi_{\epsilon}^{\left\{t_{1}\right\}}} P_{\epsilon \mid x_{\pi(i)}}^{t_{1}} h_{0}\right)\right)(y) d y \\
= & \int_{B_{1}\left(x_{\Pi(i)}\right)} h_{1}(y) d y
\end{aligned}
$$

and we will show at the end of this proof that

$$
\operatorname{var}\left(h_{1}\right) \leq \kappa K \operatorname{var}\left(h_{0}\right)
$$

for some constant $K$ (depending only on $\eta, \delta$ and $\gamma$, but not on $k$ ).

We can use this to obtain by induction an estimate for the integral appearing in (5.7), starting from $h_{0}$, the density of the normalized Lebesgue measure on $\left[\frac{d}{v}, v\right]^{U(i)}$. In the exceptional case $t_{1}=0$ the first of the three integrals in the decomposition (5.13) does not make sense, but we can use $1_{G^{c}} h_{0}=0$ instead so that we are 
left with the two other integrals only. As we only use that $h_{0} \geq 0$, but not that $\int h_{0}(y) d y=1$, we can reproduce the same argument for $h_{1}$, with $t_{2} \geq 1$ (since the times in $\tilde{V}_{\{i\}}$ have been taken at distance at least 2), obtaining $h_{2}$ such that

$$
\int_{B_{0}\left(x_{\Pi(i)}\right)} h_{0}(y) d y \leq \int_{B_{1}\left(x_{\Pi(i)}\right)} h_{1}(y) d y \leq \int_{B_{2}\left(x_{\Pi(i)}\right)} h_{2}(y) d y
$$

and $\operatorname{var}\left(h_{2}\right) \leq \kappa K \operatorname{var}\left(h_{1}\right) \leq(\kappa K)^{2} \operatorname{var}\left(h_{0}\right)$. Inductively, we obtain densities $h_{1}, h_{2}, \ldots, h_{\# \tilde{V}_{\{i\}}}$ such that

$$
\begin{aligned}
\int_{B_{0}\left(x_{\Pi(i)}\right)} h_{0}(y) d y & \leq \int_{I^{U(i)}} h_{\# \tilde{V}_{\{i\}}}(y) d y \leq \frac{1}{2} \operatorname{var}\left(h_{\# \tilde{V}_{\{i\}}}\right) \\
& \leq(\kappa K)^{\# \tilde{V}_{\{i\}}} \frac{1}{2} \operatorname{var}\left(h_{0}\right) \leq \Delta^{8 \cdot \# \tilde{V}_{\{i\}}}
\end{aligned}
$$

if $\kappa$ is chosen small enough (by taking $k$ large enough, independently of $K$ ) that $\kappa K \frac{1}{2} \operatorname{var}\left(h_{0}\right) \leq \Delta^{8}$. Inserted into (5.7) this yields (5.6).

To obtain (5.14), we notice that the three terms in the sum have the same structure

$$
\int_{B_{1}\left(x_{\Pi(i)}\right)} P_{\hat{T}} P_{A_{1}}\left(1_{G^{c}} P_{\hat{T}} P_{A_{2}} h_{0}\right)(y) d y
$$

with $P_{A_{1}}$ and $P_{A_{2}}$ representing operators (depending on $t_{1}$ and $x_{\Pi(i)}$ ) which are integral preserving and uniformly (in $t_{1}$ and $\boldsymbol{x}_{\Pi(i)}$ ) bounded in variation norm, see (5.3). As $G^{c}$ is the union of a finite number of hyper-rectangles in $I^{U(i)}$, multiplication by $1_{G_{c}}$ is a bounded linear operator with respect to the norm $\operatorname{var}($.$) .$

We denote by $K_{1}$ a uniform bound for the variation norm of $P_{A_{1}} 1_{G^{c}} P_{\hat{T}} P_{A_{2}}$ and by $K_{2}$ a uniform bound for that of $P_{A_{2}}$. One can then estimate uniformly variation and integral of $P_{A_{1}} 1_{G^{c}} P_{\hat{T}} P_{A_{2}} h_{0}$ :

$$
\operatorname{var}\left(P_{A_{1}} 1_{G^{c}} P_{\hat{T}} P_{A_{2}} h_{0}\right) \leq K_{1} \operatorname{var}\left(h_{0}\right)
$$

and, applying (2.11) to $f=P_{A_{2}} h_{0}$,

$$
\int P_{A_{1}} 1_{G^{c}} P_{\hat{T}} P_{A_{2}} h_{0} d m=\int 1_{G^{c}} P_{\hat{T}} P_{A_{2}} h_{0} d m=\int 1_{G^{c}}(h+\tilde{h}) d m
$$

with

- $\operatorname{var}(\tilde{h}) \leq \kappa \operatorname{var}(f) \leq \kappa K_{2} \operatorname{var}\left(h_{0}\right)$ so that $\int 1_{G^{c}} \tilde{h} d m \leq \frac{1}{2} \kappa K_{2} \operatorname{var}\left(h_{0}\right)$, and

- $h=\left(\int h_{0} d m\right) \hat{h}_{\alpha}$ so that, according to (2.12),

$$
\int 1_{G^{c}} h d m \leq\left(\int h_{0} d m\right) \kappa \leq \frac{1}{2} \kappa \operatorname{var}\left(h_{0}\right) .
$$

Hence we obtain

$$
\int P_{A_{1}} 1_{G^{c}} P_{\hat{T}} P_{A_{2}} h_{0} d m \leq \frac{1}{2} \kappa\left(1+K_{2}\right) \operatorname{var}\left(h_{0}\right)
$$

and estimates (5.16) and (5.17) allow us to apply (2.10) to obtain

$$
\operatorname{var}\left(P_{\hat{T}} P_{A_{1}} 1_{G^{c}} P_{\hat{T}} P_{A_{2}} h_{0}\right) \leq \kappa\left(K_{1}+\frac{1}{2}\left(1+K_{2}\right) \beta\right) \operatorname{var}\left(h_{0}\right)
$$

which implies (5.14) with $K=3\left(K_{1}+\frac{1}{2}\left(1+K_{2}\right) \beta\right)$. 


\section{Phase transition versus Bifurcation}

Proof of Theorem 2 Now $\Lambda=(\mathbb{Z} / L \mathbb{Z})^{2}$ so that $T_{\epsilon}$ is a piecewise affine, piecewise expanding self map of the $L^{2}$-dimensional cube $[-1,1]^{\Lambda}$. The existence of a $T_{\epsilon^{-}}$ invariant probability measure $d \mu_{\epsilon, \Lambda}=h_{\epsilon, \Lambda} d m^{\Lambda}$ follows rather immediately from Lemma 4.1] see e.g. Theorem 3.1 in [19]. Indeed, more is true: As the PerronFrobenius operator of $T_{\epsilon}$ is quasicompact, there is some iterate $T_{\epsilon}^{r}$ which is exact on each of its ergodic components. Therefore the uniqueness and mixing of $\mu_{\epsilon, \Lambda}$ can be proved following the folklore type strategy of [14. Namely, we will show:

$$
\begin{aligned}
& \text { If } B \subseteq I^{\Lambda} \text { is a measurable } T_{\epsilon}^{r} \text {-invariant set, } \\
& \text { then } B=I^{\Lambda} \text { modulo Lebesgue measure } 0 \text {. }
\end{aligned}
$$

This proves uniqueness of the invariant density and mixing of $\left(T_{\epsilon}, \mu_{\epsilon, \Lambda}\right)$ and, in view of the spectral gap of the Perron-Frobenius operator of $T_{\epsilon}$, also the exponential decay of correlations follows at once. In the sequel we will suppress to write "modulo Lebesgue measure 0", but all set inclusions will be understood in this way.

Denote by $\mathcal{J}$ the partition of $I=[-1,1]$ into maximal monotonicity intervals of $\hat{\tau}$. Then $\mathcal{J}^{\Lambda}$ is a partition of $I^{\Lambda}$ into hyperrectangles $R$ each of which is mapped by $\hat{T}$ homeomorphically onto its image. As some maximal monotone branches of $\hat{\tau}$ are only piecewise linear, also the $\left.\hat{T}\right|_{R}$ are only piecewise linear in general. Nevertheless, $\hat{T}(R)$ is a hyperrectangle for each $R \in \mathcal{J}^{\Lambda}$.

Now suppose $B$ is as in (6.1). A telescoping argument just as in 14 proposition $5]$ shows that $B$ contains some cube $Q$ of side length $\ell(Q)$. Elementary geometrical arguments (see [14 Lemma 8a]) show that $\Phi_{\epsilon} Q$ contains a cube $Q^{\prime}$ of side length at least $\frac{\epsilon}{1-\epsilon} \ell(Q) \geq \frac{1}{3} \ell(Q)$ (observe that $\epsilon \in\left[0, \frac{1}{4}\right]$ ). This cube $Q^{\prime}$ is partitioned by the partition $\mathcal{J}^{\Lambda}$ into hyperrectangles $Q^{\prime} \cap R$. As long as $Q^{\prime}$ is cut in no more than two pieces in each coordinate direction, at least one of the $Q^{\prime} \cap R$ contains a cube $Q^{\prime \prime}$ of side length at least $\frac{1}{2} \ell\left(Q^{\prime}\right)$. As we chose the parameter $k$ in Lemma 4.1 so large that $\left|\hat{\tau}^{\prime}\right| \geq 4$, this guarantees that $\ell\left(\hat{T} Q^{\prime \prime}\right) \geq 2 \ell\left(Q^{\prime}\right)$. In this way we conclude that $\hat{T}^{3} Q^{\prime}$ contains a subcube $Q^{\prime \prime \prime}$ of side length at least $8 \ell\left(Q^{\prime}\right) \geq \frac{8}{3} \ell(Q)$, provided that by none of the three iterated applications of $\hat{T}$ any side of the cube is cut into more than two pieces.

If, on the other hand, at any of the three stages some sides are cut into more than two pieces, then one of these pieces is a maximal monotonicity interval of $\hat{\tau}$, and as we saw in the proof of Lema 2.1 its image has length at least $\frac{u-c}{v}$. Hence, it is still guaranteed that $T_{\epsilon} Q \supseteq \hat{T}^{3} Q^{\prime}$ contains a cube $Q^{\prime \prime \prime}$ with $\ell\left(Q^{\prime \prime \prime}\right) \geq \min \left\{\frac{8}{3} \ell(Q), \frac{u-c}{v}\right\}$. Continuing in this way, $T_{\epsilon}^{n r} Q$ will contain a cube of side length at least $\frac{u-c}{v}$ for some $n>0$. As $Q \subset B=T_{\epsilon}^{r} B$, we conclude that $B$ contains a cube of side length at least $\frac{u-c}{v}$, and so $\Phi_{\epsilon} B$ contains a cube, call it $\hat{Q}$, with $\ell(\hat{Q}) \geq \frac{u-c}{3 v}$

Now, if $k$ is chosen large enough, then each monotonicity interval of $\hat{\tau}=\frac{1}{v} \tilde{\tau}^{k}$ has length less than $\frac{u-c}{6 v}$. Therefore each side of $\hat{Q}$ contains at least one maximal monotonicity interval of $\hat{\tau}$, and it follows from Lemma 2.1 that $T_{\epsilon} B=\hat{T}^{3} \Phi_{\epsilon} B \supseteq$ $\hat{T}^{3} \hat{Q}=I^{\Lambda}$. Hence $B=T_{\epsilon}^{r} B=T_{\epsilon}^{r-1} T_{\epsilon} B=T_{\epsilon}^{r-1} I^{\Lambda}=I^{\Lambda}$, i.e. 6.1). This proves at the same time that the invariant measure is indeed equivalent to Lebesgue measure.

\section{The PHASE TRANSITION FOR SMOOTH EXPANDING CIRCLE MAPS}

In this section we describe how to modify our construction such that $\tau$ becomes a smooth expanding circle map. We follow essentially the strategy from 21] where 
a piecewise linear map, whose Perron-Frobenius operator had particular spectral properties, was approximated by an analytic map in such a way that the spectal properties were essentially conserved. Our situation is different from the one there in so far as the map $\tau$ has non-surjective monotone branches, so a little modification of the strategy from 21$]$ is necessary.

The construction of the modification $\bar{\tau}$ of $\hat{\tau}$

Denote by $\dot{\tau}$ the modification of $\tilde{\tau}$ with only increasing linear branches as discussed in Remark 2.3(A] . So Lemma 2.2 holds as well for the map $\check{\tau}:=\frac{1}{v} \tau^{k}$. Let $-1=$ $a_{0}<a_{1}<\cdots<a_{p}=1$ be the partition of $[-1,1]$ into maximal monotonicity intervals of $\check{\tau}$. We are going to define an increasing, piecewise linear, continuous function $\zeta: \mathbb{R} \rightarrow \mathbb{R}$ in terms of the inverse branches of $\check{\tau}$ : for $k=0, \ldots, p-1$ and $x \in(2 k-1,2 k+1)$ let

$$
\zeta(x)= \begin{cases}a_{k} & \text { if } x-2 k \leq \check{\tau}\left(a_{k}^{+}\right) \\ \left.\check{\tau}\right|_{\left(a_{k}, a_{k+1}\right)} ^{-1}(x-2 k) & \text { if } \check{\tau}\left(a_{k}^{+}\right)<x-2 k<\check{\tau}\left(a_{k+1}^{-}\right) \\ a_{k+1} & \text { if } x-2 k \geq \check{\tau}\left(a_{k+1}^{-}\right) .\end{cases}
$$

Obviously $\zeta$ extends continuously to a (not strictly!) increasing map from $[-1,2 p-$ 1] onto $[-1,1]$. As $\zeta(-1)=a_{0}=-1$ and $\zeta(2 p-1)=a_{p}=1$, it further extends to a continuous increasing map $\zeta: \mathbb{R} \rightarrow \mathbb{R}$ by

$$
\zeta(x+2 p)=\zeta(x)+2 .
$$

Let $\varphi_{\sigma}: \mathbb{R} \rightarrow[0, \infty)$ be the Gaussian density with mean 0 and variance $\sigma^{2}$. We use it as a convolution kernel for defining

$$
\zeta_{\sigma}(x):=\zeta * \varphi_{\sigma}(x)-\zeta * \varphi_{\sigma}(-1)-1 .
$$

Obviously, $\zeta_{\sigma}(-1)=-1, \zeta_{\sigma}(x+2 p)=\zeta_{\sigma}(x)+2, \zeta_{\sigma}^{\prime}(x+2 p)=\zeta_{\sigma}^{\prime}(x)$, and as $\zeta$ is continuous and piecewise linear, $\left\|\zeta_{\sigma}-\zeta\right\|_{\infty} \leq$ const $\cdot \sigma$ and $\lim _{\sigma \rightarrow 0} \zeta_{\sigma}^{\prime}(x)=$ $\zeta^{\prime}(x)$ at all points $x$ where $\zeta$ is differentiable. Furthermore, $\inf _{x} \zeta_{\sigma}^{\prime}(x)>0$ for each $\sigma>0$. Hence $\zeta_{\sigma}^{-1}$ projects to a $p$-fold covering circle map $\check{\tau}_{\sigma}$ of $\mathbb{R} /(2 \mathbb{Z}+1)$ onto itself. In this way we are nearly in the same situation as in 21 except that $\inf _{\sigma>0} \inf _{x} \zeta_{\sigma}^{\prime}(x)=\inf _{x} \zeta^{\prime}(x)=0 .{ }^{6}$ The Perron-Frobenius operator of $\check{\tau}_{\sigma}$ can be conveniently expressed in terms of $\zeta_{\sigma}$ : for $x \in[-1,1)$,

$$
P_{\check{\tau}_{\sigma}} f(x)=\sum_{k=0}^{p-1} f\left(\zeta_{\sigma}(x+2 k)\right) \cdot \zeta_{\sigma}^{\prime}(x+2 k) .
$$

\section{Proof of Theorem 1 for the map $\bar{\tau}^{3}$}

Obviously, $\lim _{\sigma \rightarrow 0} P_{\check{\tau}_{\sigma}} f(x)=P_{\check{\tau}} f(x)$ at all points $x$ where $f$ is continuous and $\zeta$ is differentiable. Hence, although $\check{\tau}$ has many non-surjective branches whereas $\check{\tau}_{\sigma}$ has only full branches, $P_{\check{\tau}_{\sigma}}$ is "close" to $P_{\check{\tau}}$. Below we state this more precisely: we

\footnotetext{
${ }^{6}$ Our maps $\zeta$ and $\zeta_{\sigma}$ play the roles of the maps $\tau$ and $\tau_{\delta}$ from 21 section 3]. Note also that we are dealing with maps of $[-1,1]$ whereas 21 considers maps of $[0,1]$ or $\mathbb{R} / \mathbb{Z}$. We also take this opportunity to correct two misprints in 21. The first one is obvious: above eq. (2) one has $\dot{\tau}_{\delta}(x+p)$ instead of $\dot{\tau}_{\delta}(x+1)$. The second one concerns eq. (3), which is not correct as stated. It should be $\left\|2 g_{\delta, M}\right\|_{\infty}^{1 / M} \leq 2^{1 / M} \cdot\|g\|_{\infty}$, and in the line thereafter one must restrict to $\|g\|_{\infty}<\kappa<1$ instead of $\vartheta<\kappa<1$. This does not affect the main result of [21] because, for the particular map studied there, $\vartheta=\|g\|_{\infty}$. Also for the purposes of the present paper this weaker form of eq. (3) is suficient.
} 
will show that, for small enough $\sigma>0$, the Perron-Frobenius operator of $\bar{\tau}:=\check{\tau}_{\sigma}$ is only a small modification of that of $\check{\tau}$ in the sense discussed in Remark [2.3(B), namely: there are constants $\sigma_{0}, \kappa, F, C_{0}>0$ such that, for all $f:[-1,1] \rightarrow \mathbb{R}$ of bounded variation, for all $\sigma \in\left(0, \sigma_{0}\right)$ and for all $n \in \mathbb{N}$,

$$
\begin{gathered}
\operatorname{var}\left(P_{\tilde{\tau}_{\sigma}}^{n} f\right) \leq F \cdot\left(\kappa^{n} \operatorname{var}(f)+\int|f| d m\right), \\
\int\left|P_{\check{\tau}_{\sigma}} f-P_{\check{\tau}} f\right| d m \leq C_{0} \cdot \sigma \cdot \operatorname{var}(f) .
\end{gathered}
$$

Hence Theorem [1]also holds for the single-site map $\tau=\bar{\tau}^{3}$ (see Remark 2.3] (B)).

The uniform Lasota-Yorke type estimate (7.4) is proved exactly as in 21] (which is based in turn on Rychlik's approach [26]). ${ }^{7}$ The proof of (7.5) is a bit different from that in [21, because, other than in that reference, $\inf _{\sigma>0} \inf _{x} \zeta_{\sigma}^{\prime}(x)=$ $\inf _{x} \zeta^{\prime}(x)=0$. Denote by $\chi$ the indicator function of an interval $[-1, x]$. As in [21. Proposition 2] it suffices to show that $\int\left|P_{\check{\tau}_{\sigma}} \chi-P_{\tilde{\tau}} \chi\right| d m \leq C_{0} \sigma$. But $\lim _{s \rightarrow 0} P_{\check{\tau}_{s}} f(x)=P_{\check{\tau}} f(x)$ at all but at most countably many $x$, so $\lim _{s \rightarrow 0} \int \mid P_{\check{\tau}_{s}} \chi-$ $P_{\tilde{\tau} \chi} \mid d m=0$. Therefore the following estimate, which is uniform in $s \in(0, \sigma)$, completes the proof of (7.5):

$$
\begin{aligned}
& \int\left|P_{\check{\tau}_{\sigma}} \chi-P_{\check{\tau}_{s}} \chi\right| d m \\
\leq & \int_{-1}^{1} \sum_{k=0}^{p-1}\left|\chi\left(\zeta_{\sigma}(y+2 k)\right) \cdot \zeta_{\sigma}^{\prime}(y+2 k)-\chi\left(\zeta_{s}(y+2 k)\right) \cdot \zeta_{s}^{\prime}(y+2 k)\right| d y \\
= & \int_{-1}^{2 p-1}\left|\chi\left(\zeta_{\sigma}(y)\right) \cdot \zeta_{\sigma}^{\prime}(y)-\chi\left(\zeta_{s}(y)\right) \cdot \zeta_{s}^{\prime}(y)\right| d y \\
\leq & 2\left\|\zeta_{\sigma}-\zeta_{s}\right\|_{\infty}+\int_{-1}^{2 p-1}\left|\zeta_{\sigma}^{\prime}(y)-\zeta_{s}^{\prime}(y)\right| d y
\end{aligned}
$$

and both integrals are bounded by const $\cdot \sigma$ - the first one, as remarked above, because $\zeta$ is Lipschitz continuous, and the second one because $\zeta^{\prime}$ is of bounded variation.

\section{Proof of Theorem 2 for the map $\bar{\tau}^{3}$}

Let $\bar{\tau}=\check{\tau}_{\sigma}$ with a sufficiently small but fixed $\sigma>0$ be as before. Although the branches of $\bar{\tau}$ are not linear, the uniform expansion in conjunction with the bounded second derivative of $\bar{\tau}$ yields uniform distortion control on the branches of all iterates $\bar{\tau}^{n}, c f$. [13, Lemma 5.1.18]. This allows the same telescoping argument as in section [6 leading to a cube $Q$ as in that proof. The rest of the proof goes through exactly as in that section. Indeed, the argument becomes even much simpler, because now all monotone branches of the single-site map are surjective.

\section{REFERENCES}

[1] V. Baladi, Positive transfer operators and decay of correlations, Advanced Series in Nonlinear Dynamics, Volume 16, World Scientific (2000).

\footnotetext{
${ }^{7}$ As in our case $\check{\tau}^{\prime} \geq 4$, one can make the following simplifying choices in 21 section 2]: $N=M=1, \epsilon=\frac{1}{4}, \lambda_{1}=\kappa=\frac{3}{4}$ and $D_{1}=2 / \min _{A \in \alpha} m(A)$ if $\sigma>0$ is small enough, where the partition $\alpha=\alpha_{1}$ is obtained from the partition into maximal monotonicity intervals of $\check{\tau}$ by cutting each of these intervals into two subintervals with equal length, and finally $F=4 D_{1}$.
} 
[2] V. Baladi, M. Degli Eposti, S. Isola, E. Järvenpää, A. Kupiainen, The spectrum of weakly coupled map lattices, J. Math. Pures Appl. 77 (1998), 539-584.

[3] J.-B. Bardet, Limit theorems for coupled analytic maps, Probab. Th. Rel. Fields 124 (2002), 151-177.

[4] C. Boldrighini, L.A. Bunimovich, G. Cosimi, Frigio, A. Pellegrinotti, Ising-type and other transitions in one-dimensional coupled map lattices with sign symmetry, J. Stat. Phys. 102 (2001), 1271-1283.

[5] R. Bowen, Bernoulli maps of the unit interval, Israel J. Math. 28, 161-168 (1977).

[6] J. Bricmont, A. Kupiainen, Coupled analytic maps, Nonlinearity 8 (1995), no. 3, 379-396.

[7] J. Bricmont, A. Kupiainen, High temperature expansions and dynamical systems, Comm. Math. Phys. 178 (1996), no. 3, 703-732.

[8] L.A. Bunimovich, Ya.G. Sinai, Space-time chaos in coupled map lattices, Nonlinearity 1 (1988), 491-516.

[9] T. Fischer, H.H. Rugh, Transfer operators for coupled analytic maps, Ergod. Th.\& Dynam. Sys. 20 (2000), 109-143.

[10] G. Gielis, R. MacKay, Coupled map lattices with phase transitions, Nonlinearity 13 (2000), 867-888.

[11] M. Jiang, Sinai-Ruelle-Bowen measures for lattice dynamical systems, J. Statist. Phys. 111 (2003), no. 3-4, 863-902.

[12] W. Just, F. Schmüser, On phase transitions in coupled map lattices, in: Dynamics of Coupled Map Lattices and of Related Spatially Extended Systems (Eds.: J.-R. Chazottes, B. Fernandez), Lecture Notes in Physics 671 (2005), pp. 33-64, Springer Verlag.

[13] A. Katok, B. Hasselblatt, Introduction to the Modern Theory of Dynamical Systems, Encyclopedia of Mathematics and its Applications, Volume 54, Cambridge University Press (1995).

[14] G. Keller, Mixing for finite systems of coupled tent maps, Proc. Steklov Inst. Math. 216(1997), 315-321. (Also: Erwin-Schrödinger-Institut Preprint ftp://ftp.esi.ac.at/pub/Preprints/esi388.pdf)

[15] G. Keller, An ergodic theoretic approach to mean field coupled maps, in: Fractal Geometry and Stochastics II (Eds.: C. Bandt, S. Graf, M. Zähle), Progress in Probability, Vol. 46 (2000), pp. 183-208, Birkhäuser.

[16] G. Keller, M. Künzle, Transfer operators for coupled map lattices, Ergod. Th.\& Dynam. Sys. 12, 297-318 (1992)

[17] G. Keller, C. Liverani, Stability of the spectrum for transfer operators, Annali della Scuola Normale Superiore di Pisa, Scienze fisiche e Matematiche, (4) XXVIII, 141-152 (1999).

[18] G. Keller, C. Liverani, Coupled map lattices without cluster expansion, Discrete and Continuous Dynamical Systems, 11, n.2,3, 325-335 (2004).

[19] G. Keller, C. Liverani, A spectral gap for a one-dimensional lattice of coupled piecewise expanding interval maps, in: Dynamics of Coupled Map Lattices and of Related Spatially Extended Systems (Eds.: J.-R. Chazottes, B. Fernandez), Lecture Notes in Physics 671 (2005), pp. 115-151, Springer Verlag.

[20] G. Keller, C. Liverani, Uniqueness of the SRB measure for piecewise expanding weakly coupled map lattices in any dimension, Commun. Math. Phys. 262, 33-50 (2006).

[21] G. Keller, H.H. Rugh, Eigenfunctions for smooth expanding circle maps, Nonlinearity $\mathbf{1 7}$ (204), 1723-1730.

[22] G. Keller, R. Zweimüller, Unidirectionally coupled interval maps: between dynamics and statistical mechanics, Nonlinearity 15, 1-24 (2002)

[23] J.L. Lebowitz, C. Maes, E.R. Speer, Statistical mechanics of probabilistic cellular automata, J. Stat. Phys. 59 (1990), 117-170.

[24] R. MacKay, Incecomposable coupled map lattices with non-unique phase, in: Dynamics of Coupled Map Lattices and of Related Spatially Extended Systems (Eds.: J.-R. Chazottes, B. Fernandez), Lecture Notes in Physics 671 (2005), pp. 65-94, Springer Verlag.

[25] H.H. Rugh, Coupled maps and analytic function spaces, Ann. Sci. École Norm. Sup. (4) 35 (2002), no. 4, 489-535.

[26] M. Rychlik, Bounded variation and invariant measures, Studia Math. LXXVI (1983), 69-80.

[27] A.L. Toom, Stable and attractive trajectories in multicomponent systems, in: Multicomponent Random Systems (Eds.: R.L. Dobrushin, Ya.G. Sinai), Dekker, New York (1980). 
J.-B. Bardet, IRMaR/UfR Mathématiques, Université de Rennes 1, Campus de Beaulieu, 35042 Rennes Cedex, France; G. Keller, Mathematisches Institut, Universität ErlangenNÜrnberg, Bismarckstr. 1 1/2, 91054 Erlangen, Germany

E-mail address: jean-baptiste.bardet@univ-rennes1.fr, keller@mi.uni-erlangen.de 